\title{
Regulation of biotic and abiotic stress responses by plant hormones
}

\author{
Prakash P. Kumar
}

Received: 28 April 2013/Accepted: 18 May 2013/Published online: 29 May 2013

(c) Springer-Verlag Berlin Heidelberg 2013

This is the second special issue on Plant Hormone Signaling. Abscisic acid (ABA), ethylene and salicylic acid (SA) have long been recognized as the key plant hormones mediating abiotic and biotic stresses, respectively. The underlying mechanisms of action by which these and other hormones modulate the response of plants to stresses have received considerable attention in the recent past. Research findings in the last two decades have been revealing their fascinating modes of action. Furthermore, the intricate crosstalks among various hormones by which they can modulate growth and development in response to diverse environmental stresses have emerged as a common theme in this field.

With the identification of specific receptors for individual hormones, convincing mechanisms explaining the modes of action of these hormones have been proposed. The involvement of ethylene and the AP2/ERF family of proteins, PR proteins and the like in biotic stress response has now been well established. Besides ABA and ethylene, it is now known that salicylic acid, jasmonates, brassinosteroids, and even gibberellins and auxins crosstalk extensively to regulate practically all aspects of plant stress responses.

Eminent practitioners in the field have provided enthusiastic support for this issue as with the first special issue, which dealt with general aspects of hormone signaling. The sixteen contributions compiled in this issue are articles dealing specifically with abiotic and biotic stresses. The contributions range from how hormones initiate signal cascades to control grain yield, root development, and DNA repair as well as protein modifications such as SUMOylation. Also included are papers on the crosstalks among ABA, ethylene, gibberellins, brassinosteroids, SA, jasmonates etc. to regulate herbivory and other biotic stresses. It is hoped that the timely reviews of the signaling-related topics in the two special issues will be a useful contribution to plant biologists in general.

Exciting new findings continue to be made, and work on plant hormone signaling intermediates holds tremendous promises for introducing new traits and protecting high levels of crop productivity in the face of global climate change. We can be confident in declaring that the proposed modes of action of plant hormones will continue to get refined and such studies will help in boosting crop productivity in the coming decades. Hopefully, this effort to put together a collection of useful reference material will be appreciated by our readers.

Acknowledgments I wish to record my sincere thanks to all the contributors and the people at the Editorial office of the journal for their excellent cooperation, which made it possible to complete this challenging task. It would not have been possible for me to coordinate assembling the two special issues in a relatively short time without such wonderful support.

Communicated by G. Hahne.

P. P. Kumar $(\bowtie)$

Department of Biological Sciences, National University of

Singapore, 10 Science Drive 4, Singapore 117543, Singapore

e-mail: dbskumar@nus.edu.sg 